\title{
INVESTIGATIONS ON THE INTERNAL CALIBRATION OF MULTI-CHANNEL SAR
}

\author{
M. Younis, T. Rommel, F. de Almeida, S. Huber, M. Martone, M. Villano, and G. Krieger
}

German Aerospace Center (DLR), Oberpfaffenhofen, Germany

\begin{abstract}
A calibration scheme for future SAR systems utilizing digital beamforming, is suggested. These systems pose new challenges for the calibration, as the increased complexity of multi-channel SAR does not allow for the extrapolation of current calibration techniques. Further, depending on the operation mode, real-time error measurement and correction is unavoidable, which requires a reconsideration to current calibration approaches. On the other hand, the digital hardware already present in multi-channel SAR systems offer new possibilities for novel calibration such as on-board error correction and digital calibration. This paper presents a calibration scheme suitable for multi-channel digital beamforming SAR.
\end{abstract}

Index Terms - Calibration, Multi-Channel Calibration, Internal SAR Calibration, SAR Internal Errors

\section{DIGITAL BEAM-FORMING SAR CONCEPT}

A multi-channel SAR instrument utilizing digital beamforming is considered for the calibration concept being descirbed in this paper. Here, a general overview of the functionality of such an instrument is given, while the specific technical implementation given in $[1,2]$. The planar direct radiating SAR antenna consists of $N_{a z} \times N_{e l}$ antenna elements in along-track (azimuth) and across-track (elevation) direction, respectively, where each antenna element is equipped with Transmit/Receive Modules (TRM).

On-transmit, all the TRMs are active, i.e. transmitting; to get an antenna beam wider than what would be the case for a large antenna, phase spoiling is applied. The reflected echo signal is received by the antenna elements, amplified, and then $N_{\text {comb }}$ TRM outputs are combined, where $N_{\text {comb }}$ typically consists of 1 to 2 antenna elements in azimuth and 2 to 8 elements in elevation. The resulting $N_{a z} \cdot N_{e l} / N_{c o m b}$ signals are filtered, down-converted and digitized, forming $n_{a z} \times n_{e l}$ azimuth and elevation data streams, respectively.

Digital beam-forming is first applied through a technique known as Scan-On-REceive (SCORE) [3]. It is applied in elevation by multiplying the $n_{e l}$ data streams of each azimuth channel by time varying complex weights, and by

The work is carried out under a contract with the European Space Agency (ESA/ESTEC) ESA ITT AO/1-8469/15/NL/FE this forming a time varying beam which follows the SAR signal echo while it traverses the ground. After SCORE a total of $n_{a z}$ data streams remain which are stored in memory and later down-linked to the ground ${ }^{1}$. On-ground a second digital-beamforming is applied to reconstruct the unambiguous Doppler spectra from the azimuth data [5], before proceeding to a-more or less- conventional SAR processing.

\section{CALIBRATION CONCEPT}

The main characteristic of the calibration concept described next, is that it avoids interrupting SAR operation - which would cause lost imaging pulses, and, that it considers onboard channel correction. In the following the calibration scheme for each of the instrument units is reviewed [1].

\subsection{Transmit/Receive-Module Calibration Scheme}

Consider first the RF instrument containing the TRMs as shown in Fig. 1. For the transmit path calibration (mainly HPA drift) part of the transmitted (chirp) signal power is coupled out after the circulator and fed into the receiver path through the calibration network; this characterizes (measures) of the transmit and the receive path over the full chirp bandwidth.

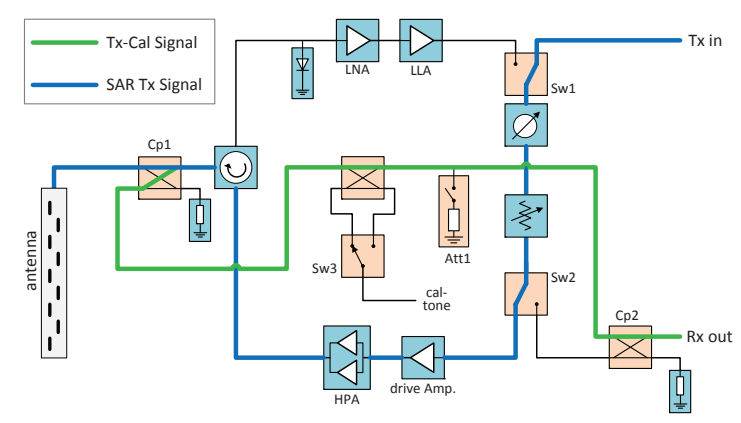

Fig. 1. Schematic of TRM showing the Tx SAR signal path coupled into the receiver ( $\mathrm{Tx}-\mathrm{Cal})$.

Injected calibration signals are, on the other hand, single tone (i.e. single frequency) signals outside the SAR chirp

\footnotetext{
${ }^{1}$ In some cases, e.g. [4], additional on-board processing of the azimuth channel data may be necessary mainly for data reduction
} 
bandwidth, which can thus co-exist with the chirp and echo signal without disturbing SAR operation [6]. As shown in Fig. 2 a separate injection point, which is different from the Tx-signal input, is chosen for the cal-tone. The TRMs allow routing the cal-tone through two different paths (compare Fig. 2 and Fig. 3). The Short-Cal path is used to measure the receive path excluding the TRM contribution; the use of this is twofold, for one it measures and removes the $\mathrm{Rx}$ path contribution from the Tx-Cal (note that the Tx-Cal is wanted to measure transmit path, i.e. mainly the HPA drift, however what is actually measured is the transmit and receive path). Second, the amplitude and phase drifts measured through the Short-Cal need to be corrected (in real time) for proper SCORE operation. As such, the Short-Cal measurement is utilized both during the transmit and the receive path calibration; Short-Cal can be simultaneous to the Tx-Cal or replacing an $\mathrm{Rx}-\mathrm{Cal}$.

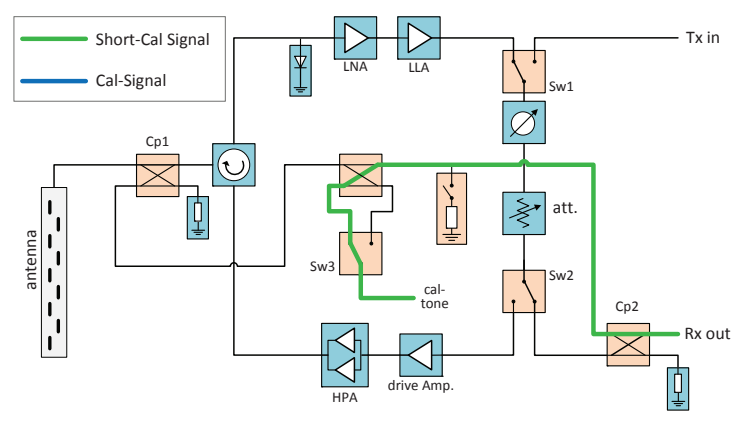

Fig. 2. Schematic of TRM showing the cal-tone injection for a Short-Cal measurement.

Finally, to characterize the receive path of the TRMs the cal-tone is routed through the output coupler into the receive path including the LNA, phase shifter, attenuattor (analog phase/amplitude setting), and the remaining Rx path, as shown in Fig. 3. The contribution of the latest is, again, removed using the Short-Cal measurement.

As mentioned above, since the cal-tone is outside the chirp bandwidth, it can be injected simultaneously to transmit or receive events without affecting them. Note that the cal-tone is not used to characterize the Tx path, thus avoiding spurious transmission of the amplified cal-tone. The evaluation of the cal-tone requires separating the same from the chirp signals; this is done in the digital domain using an FIR filter thus utilizing the processing capabilities of the FPGAs. The phase and amplitude of the cal-tone needs to be measured in real-time, since it contains the information on the imbalances of the channels. As these are single-frequency signals, the processing capability can easily be included in the FPGAs; the signals being either compared to a common digital reference, or to each other through interconnections between the FPGAs. The advantage of the cal-tone in terms of simplicity and simultaneity has the disadvantage of offering only two frequency measurement points below and above the actual chirp spectrum. Keeping in mind, that the purpose is to measure channel drifts (i.e. changes in the frequency transfer function) which typically do not alter the shape of the spectrum, suggests that the cal-tone approach is perfectly valid. Further, the raw data calibration, introduced later, allows characterizing the channels versus frequency.

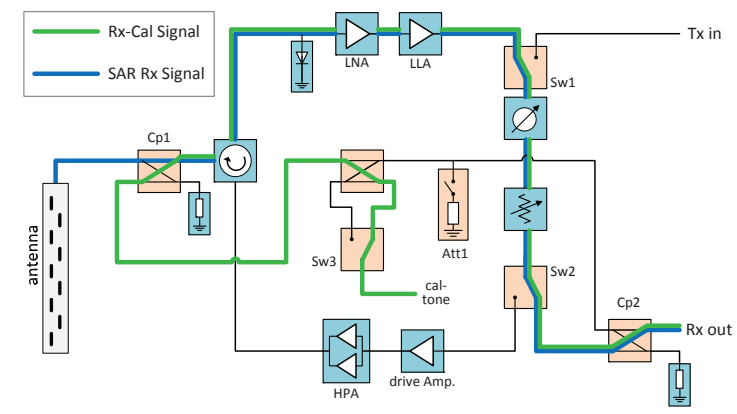

Fig. 3. Schematic of TRM showing the cal-tone injection for measurement of the receive path $(\mathrm{Rx}-\mathrm{Cal})$.

\subsection{Raw Data Based Calibration}

The above calibration concept is similar to state-of-the-art internal SAR calibration concepts in that errors beyond the output coupler (Cp1 in Fig. 3), e.g. at the antenna, are not measured and are thus unknown. These and other deficiencies addressed in [7] are overcome by using data driven calibration, meaning that the channel errors are extracted from the (raw) receive SAR data. Data driven calibration algorithm are typically based on the correlation between the receive signals of different channels and some metric to extract the errors. For example, the method in [8] shows that the correlation between the signals of any two antenna elements (spatial correlation) depends only on the separation between the two elements. The underlying model assumes the signals to originate from non-coherent scatterers, i.e. the typical raw echo signal (clutter) of a radar imaging a distributed scene. Other algorithms are available in literature, such as [9] which uses an approach based on maximizing the contrast.

Data driven calibration is not suitable for transmit path characterization $^{2}$. On the other hand, only passive and thus reciprocal RF hardware contributes to drifts beyond the couple, these contributions can be removed as they are known from the $\mathrm{Rx}$ data driven calibration Most data driven calibration techniques are suitable for correcting slowly varying errors. This is because of the computational load and the number of independent measurements needed. This is also true for the technique described here, which is understood to compensate a slow instrument drifts.

\footnotetext{
${ }^{2}$ The calibration scheme suggested here re-injects the Tx signal into the Rx path would thus technically allow the use of data driven calibration, however obviously this would be useless.
} 
Simulations were used to estimate the accuracy of data driven calibration. A random uniform phase errors is added to the simulated signals received by the antenna elements in addition to an additive Gaussian noise resulting in a $10 \mathrm{~dB}$ signal-to-noise ratio at each element. A qualitative assessment of the calibration accuracy is shown in Fig. 4 which represents the residual phase error (i.e. the phase error in the estimating the phase error) following the algorithm in [8]. The value on the abscissa represents the separation between the antenna elements whose signals are being correlated; the left most values results from comparing pairs of adjacent elements. The residual error is very small when the correlation between adjacent elements is used, increasing the separation reduces the correlation and by this increases the residual error. A quantitative analysis of the residual error shows that its value is below $1^{\circ}$ for separations of up to six elements.

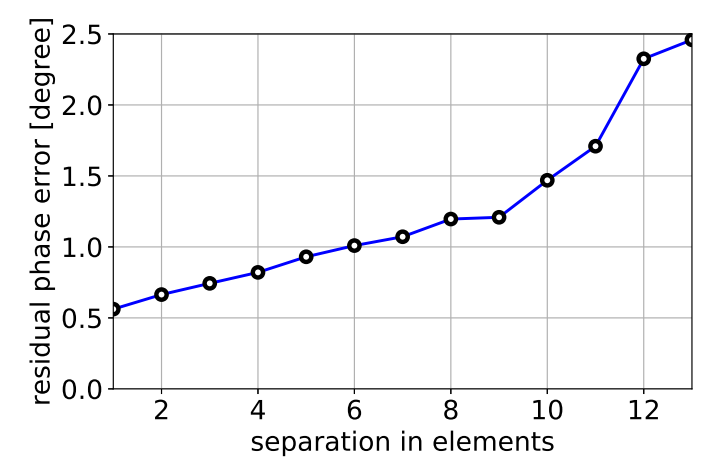

Fig. 4. Residual phase error after applying raw data based calibration to estimate the channels' phase error

\subsection{Radio Frequency Unit Calibration Scheme}

The radio frequency unit (RFU) shown in Fig. 5 includes a power combiner, filters, and a down converter. Its output, which is the summation of $N_{\text {com }}$ signals, where, typically $1 \leq N_{\text {com }} \leq 8$ is fed to the analog-to-digital converter; each RFU corresponds to one elevation channel. The RFU calibration is crucial, since it contains filters which might show a strong temperature dependency. A particularity is the power combiner, which, in terms of determining of the individual TRM errors is undesired, as the combiners output also contains the combined (sum) error signal thus resulting in an under-determined system of equations.

The transmitted signal injected into the calibration path is fed to the power combiner. To measure an individual TRM it is necessary to suppress the signals of the other TRMs at the combiner's input. This is done either by including switches in front of the power combiner, or, preferably to avoid placing switches in the SAR signal path, include an attenuator within the TRMs calibration path (see Fig. 3). In either case the transmit path of a single TRM including the RFU will be measured.

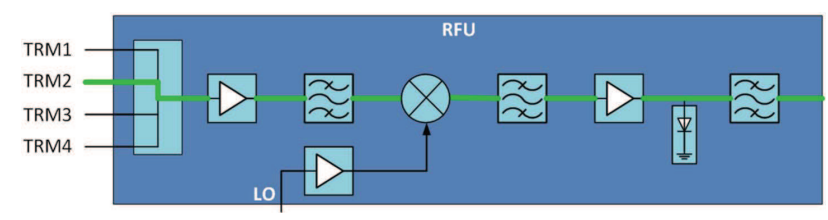

Fig. 5. Schematic of RFU showing the path of the calibration signal (Rx-Cal, Tx-Cal or Sort-Cal) from TRM 2.

For Rx-Cal, shown in Fig. 5 for $N_{c o m}=4$, one out of $N_{\text {com }}$ cal-tones are active (or two if different cal-tone frequencies are used) and will be routed through the RFU. Thus although the SAR echo signals of all TRMs are fed to the RFU it is only the Rx path of one TRM in every elevation channel which is being characterized. Consequently, if the active caltone is changed at the PRF rate, it will require $N_{c o m} / f_{P R F}$ seconds to measure all the TRMs of the instrument.

As mentioned before, the Short-Cal serves two purposes: to characterizing the RFU and to remove the RFU path contribution from the Tx-Cal. The same argumentation as above lead to a sequential activation of the Short-Cal tone in 1 of $N_{\text {com }}$ TRMs; which offers the interesting possibility of being able to measure drifts of the calibration network itself (used for injecting the cal-tone). This is because a difference between the $N_{\text {com }}$ Short-Cal measurements can only be caused by a difference in the calibration network path itself; as it is not expected to have a drift in the RFU during the $N_{c o m} / f_{P R F}$ seconds needed to switch the cal-tone through all TRMs. This way, the Short-Cal can be used to simplify the on-ground instrument characterization (note that this advantage would not given for a cal-tone which is injected through the nominal Tx path).

\subsection{Digital Beam-Forming Unit Calibration Aspects}

Up to now, the calibration of the instrument path up to the digital beam-forming unit (DBU) is considered. The error contribution of the digital part include the beam-forming, i.e. the multiplication of the data streams by complex weights and subsequent summation; meaning the SCORE operation mode. Deterministic error contributions (e.g. the update rate of the digital weights) or other known (digital weight quantization or load on the ADC input) [10] should not be considered in the calibration context but rather as a system design task. Other error sources depend on the specific hardware implementation of SCORE (for example digital summation or DAC-summation-ADC); these are not covered here. Instead the required functionality within the DBU is addressed in the following.

Each DBU, see schematic in Fig. 6, represents one digital channel of the system. Its input is the IF output signal of the RFU which is digitized, filtered, and multiplied by a complex (digital) time varying weight. The outputs of all DBUs within one antenna column are summed yielding a sin- 
gle data stream ${ }^{3}$. In addition, the FPGA (or ASIC) of the DBU needs to do the computations necessary to calibrate the system. This includes the extraction of the cal-tone (filter) and computing correction coefficients by comparing the cal-tone to a reference tone or to the cal-tones of the other DBUs utilizing the interconnection lines between the DBUs. The phase and amplitude correction coefficients are passed to the respective TRMs. Further, for the data driven calibration, the DBU needs to compare (a simplified correlation) the signals of the individual channels, again utilizing the DBU interconnection lines shown in Fig. 6. In addition, the DBU needs to generate calibration signals, which are passed through the subsequent stages in order to calibration the hardware implementing the SCORE (i.e. up to the CE). Last but not least, the correction of these subsequent hardware is done in-advance within the DBU.

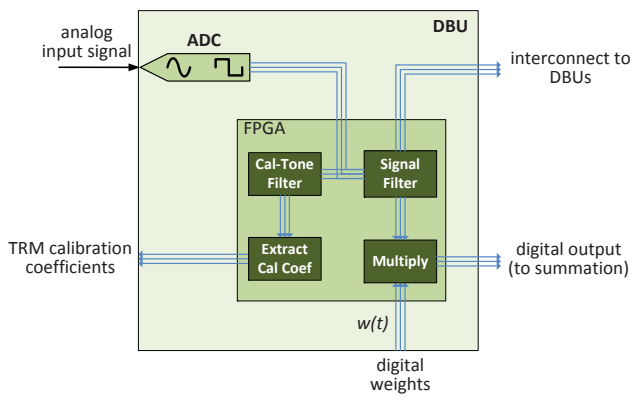

Fig. 6. Schematic DBU showing the FPGA and the various input/output lines.

\section{CONCLUSION}

Comparing the calibration concept introduced in this paper to conventional internal SAR calibration methods is not straight forward. For one, because the requirements of a multi-channel calibration, such as real time on-board capability, are rather different from what is currently being utilized. Second, because the implication of the instrument hardware, such as the requirement for an additional FIR filter for cal-tone separation are not yet fully detailed. Nevertheless, some of the main advantages of the calibration concept are worth mentioning here: it simplifies the TRM design and increases reliability by reducing the number of switches in the signal path; it allows for simultaneous SAR operation and calibration, i.e. uninterrupted SAR operation; it covers the complete SAR signal path including those not covered by the cal-signals. At the same time it should be noted that the calibration concept is of limited sensitivity to variations within the chirp bandwidth, and that the concept makes extensive use of the on-board digital processing capabilities.

\footnotetext{
${ }^{3}$ For the case of multi-SCORE operation, several data streams are output, each representing the echo signal of one pulse on the ground.
}

\section{REFERENCES}

[1] "Calibration and data reduction for digital beam forming instruments - concept discussion," European Space Agency (ESA/ESTEC), technical report D1, ESA ITT AO/18469/15/NL/FE, Mar. 2017.

[2] G. Adamiuk, C. Heer, and M. Ludwig, "DBF technology development for next generation of ESA C-Band SAR mission," in Proc. European Conference on Synthetic Aperture Radar EUSAR'2016, Hamburg, Germany, June 2016.

[3] M. Süß and W. Wiesbeck, "Side-looking synthetic aperture radar system," European Patent EP 1241 487, Sept., 2002.

[4] F. Almeida, M. Younis, G. Krieger, and A. Moreira, "Multichannel staggered SAR azimuth processing," IEEE Transactions on Geoscience and Remote Sensing, submitted 2016.

[5] G. Krieger, N. Gebert, and A. Moreira, "Unambiguous SAR signal reconstruction from non-uniform displaced phase centre sampling," IEEE Geoscience and Remote Sensing Letters, vol. 1, no. 4, pp. 260-264, Oct. 2004.

[6] J. Hoffman, S. Horst, L. Veilleux, H. Ghaemi, and S. Shaffer, "Digital calibration system enabling real-time on-orbit beamforming," in 2014 IEEE Aerospace Conference, Mar. 2014.

[7] M. Younis, C. Laux, N. Al-Kahachi, P. López-Dekker, G. Krieger, and A. Moreira, "Calibration of multi-channel spaceborne SAR - challenges and strategies," in Proc. European Conference on Synthetic Aperture Radar EUSAR'2014, Berlin, Germany, June 2014.

[8] E. H. Attia and B. D. Steinberg, "Self-cohering large antenna arrays using the spatial correlation properties of radar clutter," IEEE Transactions on Antennas and Propagation, vol. 37, pp. 30-38, Jan. 1989.

[9] G. Farquharson, P. López-Dekker, and S. J. Fraiser, "Contrastbased phase calibration for remote sensing systems with digital beamforming antennas," IEEE Transactions on Geoscience and Remote Sensing, vol. 51, pp. 1744-1754, Mar. 2013.

[10] J. P. Kroll, "Untersuchung und Modellierung von Hardwareeinflüssen auf Mehrkanal-SAR-Systeme," Master's thesis, Karlsruhe Institute of Technology (KIT), Sept. 2016. 